\title{
Treatment of Trachoma in the West Bank
}

A comparative trial of intermittent, family-based treatment of trachoma with oral doxycycline, oral sulphametopyrazine, or topical tetracycline eye ointment in two villages in the West Bank.

\author{
L. C. CHUMBLEY ${ }^{1}$, N. D. VISWALINGAM ${ }^{2}$, I. M. THOMSON ${ }^{1}$ and M. A. ZEIDAN ${ }^{1}$ \\ Jerusalem and London
}

\begin{abstract}
Summary
A double-blind stratified treatment trial was carried out on 368 patients with moderate to severe trachoma in two West Bank villages to assess the efficacy of family based therapy using tetracycline eye ointment, oral doxycycline or oral sulphametopyrazine. Treatment with tetracycline eye ointment twice daily for five days each month for six months, or a weekly dose of oral doxycycline, $5 \mathrm{mg} / \mathrm{kg}$ body weight for three weeks, or a weekly dose of oral sulphametopyrazine, $35 \mathrm{mg} / \mathrm{kg}$ body weight for three weeks was given to the patients and the members of their respective families. Clinical cure rates of $73 \%$ for tetracycline eye ointment, $\mathbf{7 4 \%}$ for doxycycline and $\mathbf{7 2} \%$ for sulphametopyrazine were achieved three months after treatment, improving to 79,80 and $82 \%$ respectively at one year. There were no statistically significant differences between the three groups treated.
\end{abstract}

Trachoma is the major cause of preventable blindness in the world, and it is a major public health problem in the Middle East. It affects about 500 million people of which at least two million are blind, and a much larger number suffer from partial loss of vision. ${ }^{1}$ In a recent survey of eye disease in the West Bank and Gaza Strip, it was found that trachoma was a significant cause of blindness in these two areas. ${ }^{2}$

Topical therapy of trachoma using tetracycline eye ointment has been proven effective $^{3}$, and it can be used as the standard against which other treatments can be compared. However, there are considerable logistical problems in delivering continuous intensive topical therapy. Intermittent therapy using tetracycline eye ointment has also been tried and has proven to be effective against trachoma. ${ }^{4}$ Oral therapy with shortacting tetracyclines and sulphonamides is also effective against trachoma, but the frequency and duration of continuous treatment, and the rate of occurrence of side effects, makes such treatment inadvisable on a mass scale. ${ }^{1}$ Studies have also shown that trachoma can be treated effectively with intermittent use of long acting tetracyclines (doxycycline) or sulphonamides (sulphametopyrazine).$^{5}$

In this report we present the results of a comparative field study, which was designed to compare the efficacy of family based intermittent therapy of hyperendemic trachoma using topical oxytetracycline eye ointment, oral doxycycline, or oral sulphametopyrazine, in two West Bank villages.

\section{Material and Methods}

Selection of patients

The whole population of the two villages of Tarqumya and Idna, in the Hebron District

Correspondence to: Sir Stephen Miller KCVO, MD, FRCS, The Order of St John, Grosvenor Crescent, London SW1X 7EF.

From the ${ }^{1}$ St. John Ophthalmic Hospital, Jerusalem and the ${ }^{21}$ Sub-department of Virology, Institute of Ophthalmology,Judd Street, London. 
in the West Bank were screened for signs of active trachoma. Only those diagnosed clinically as having moderate or severe trachoma were included in the study.

\section{Clinical examination}

The initial screening of the population for the detection of active trachoma was carried out by three ophthalmologists (LCC, NDV and IMT), using a $\times 4$ telescopic loupe and torch or a Keeler hand held slit lamp; only the upper tarsus was examined. The patients with active trachoma were re-screened and those with moderate or severe trachoma were identified by one examiner (NDV) using the hand held slit lamp. The same examiner (NDV) carried out the final screening at 3 months and one year after treatment. The severity of clinical signs were scored on a 0-3 scale and recorded separately for each eye. The overall severity of trachoma was calculated as severe, moderate or mild in accordance with a system previously described. ${ }^{6}$ Old follicles presenting as yellowish and flattened elevations, or a mild papillary response with no follicle was considered as inactive disease. Using these criteria, a clinical score of less than 10 was taken as inactive or cured.

\section{Stratified Randomisation}

The three treatment groups were stratified according to the severity of disease, size of family, number of patients with moderate to severe trachoma in each family, age and sex.

\section{Medication}

Patients were treated with one of the following regimens:

(1) Oxytetracycline eye ointment, twice daily in both eyes for five consecutive days, repeated each month for six months. The ointment was applied by mothers or an older member of the family after being taught the method of administration by a health worker, who was also responsible for a regular supply of eye ointment.

(2) Doxycycline orally $(5 \mathrm{mg} / \mathrm{kg}$ body weight), once a week for three consecutive weeks, given by a health worker

(3) Sulphametopyrazine orally $(35 \mathrm{mg} / \mathrm{kg}$ body weight), once a week for three consecutive weeks, given by a health worker.

All members of the patient's family were also treated with the same regimen, but were not included in the study. Pregnant women were treated with oxytetracycline eye ointment only.

\section{Follow-up Examination}

Follow-up examinations and photography were repeated at three months and one year following commencement of therapy. A drug questionnaire to ascertain patients' views on the effectiveness of the therapy given and any adverse effects experienced was completed by each patient included in the study.

\section{Laboratory investigation}

Conjunctival swabbings from both eyes were taken before the start of treatment. The swabbings were placed in 2SP transport medium and stored in liquid nitrogen $\left(-180^{\circ} \mathrm{C}\right)$ for transport to London for chlamydial isolation. However, inadvertent delays at local customs offices caused the specimens to remain at ambient temperature for several days.

\section{Back-up Services}

Six months after commencement of therapy, a regular mobile eye clinic service was started in both villages. All suspected bacterial eye infections encountered were treated or referred to the hospital as appropriate.

\section{Results}

Three hundred and sixty-eight patients with moderate or severe trachoma were included in the study. Of these, $189(51.4 \%)$ were females, and $179(48.6 \%)$ were males. The numbers of patients allocated to each treatment group were: tetracycline ointment 134; doxycycline 122; and sulphametopyrazine 112. The age distribution of the patients included in each treatment group is shown in Figure 1.

A follow-up rate of $100 \%$ was achieved for all 368 patients at both three months and one year. The changes in the severity of the clinical signs, observed in the upper tarsal conjunctiva in the three treatment groups, are shown in Figure 2. 
Clinical cure at three months was observed in $98(73 \%)$ patients using tetracycline ointment; $90(74 \%)$ on doxycycline and $81(72 \%)$ sulphametopyrazine. The cure rates at one year follow-up were 106 (79\%); 97 (80\%) and $92(82 \%)$ respectively. There were no statistically significant differences between the three treatments at three months and one year. All culture tests for C.trachomatis were negative because of storage of the swabbings at ambient midsummer temperature for several days.

In each treatment group, there were three patients who did not respond to treatment. Of these nine failures, seven were males and two were females, and all were younger than 21 years of age. The patients using the ointment found it cumbersome to apply, the ointment melted in the prevailing high temperature and caused blurring. Oral therapy was more popular than the eye ointment. Oral doxycycline was the easiest to administer.
Sulphametopyrazine dissolves poorly and careful supervision was needed to make sure the correct dose was taken by the patient. The entire population of both villages were re-examined at the one year follow-up. No new cases of trachoma were detected in this population.

\section{Adverse Reactions}

In the tetracycline ointment group, 32\% complained of burning, $10 \%$ of blurring of vision, and $5 \%$ of redness. In the doxycycline group, $7 \%$ complained of nausea, and two patients complained of initial transient dizziness which did not recur with subsequent doses. In the sulphametopyrazine group, $10 \%$ reported nausea, one patient and his brother (not included in the trial) experienced a rash, necessitating discontinuation of medication, and one patient reported transient dizziness which did not recur with subsequent doses.

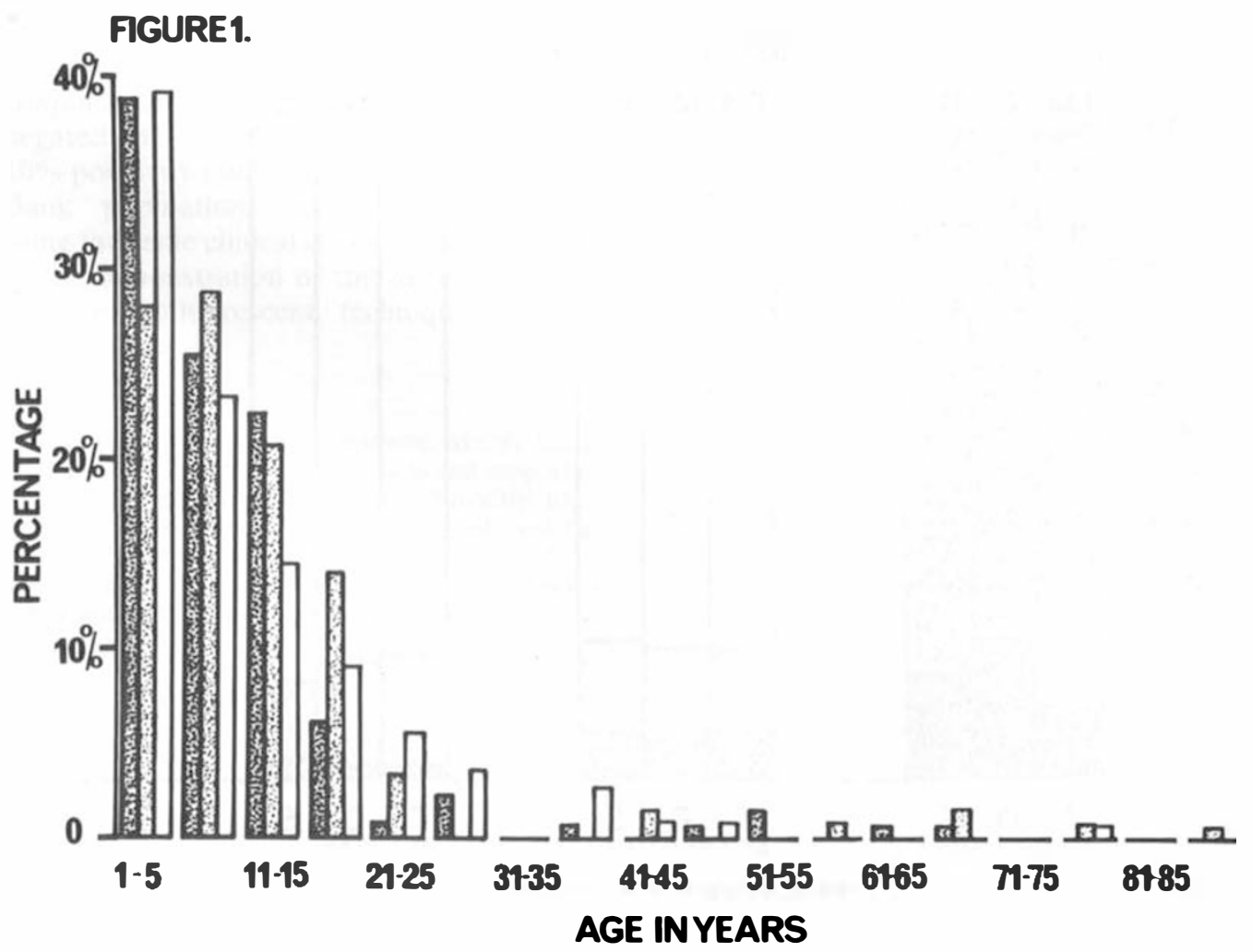

Fig. 1. Age distribution of patients with moderate to severe trachoma in present study. 
Two women (not included in the trial) had incomplete abortions within 12 hours of taking sulphametopyrazine. Prior to being given medication both had stated they were not pregnant.

\section{Discussion}

The results of this study demonstrated an overall clinical cure rate of 72 to $74 \%$ at three months, with further improvement to 79 to $82 \%$ at one year. In the remaining patients the severity of clinical signs reduced considerably.

Previous studies have shown that when the trachomatous inflammatory changes were assessed in the whole conjunctiva, the cure rates were lower than those obtained by examining the upper tarsus alone. In this study, only the upper tarsus was examined and no attempt was made to examine the whole conjunctiva because of practical difficulties working in these villages and the lack of facilities at the time. Case-based treatment studies have shown re-emergence of inflammation on later follow-up, believed to be due to re-infection by infected family members, neighbours, or playmates. ${ }^{7}$ In this study the whole family was treated in order to reduce intra-familial transmission. No new cases of trachoma were detected at the end of a year follow-up, confirming the findings of previous studies. ${ }^{3,8}$ Close supervision in giving treatments resulted in uniformly high compliance. Oral doxycycline is the easiest treatment to administer, while oral sulphametopyrazine dissolves poorly, and administration of tetracycline eye ointment is cumbersome and unpopular.

Epidemics of bacterial conjunctivis are known to play a role in the transmission of trachoma ${ }^{9}$ and in previous studies in areas where trachoma has been a blinding disease,

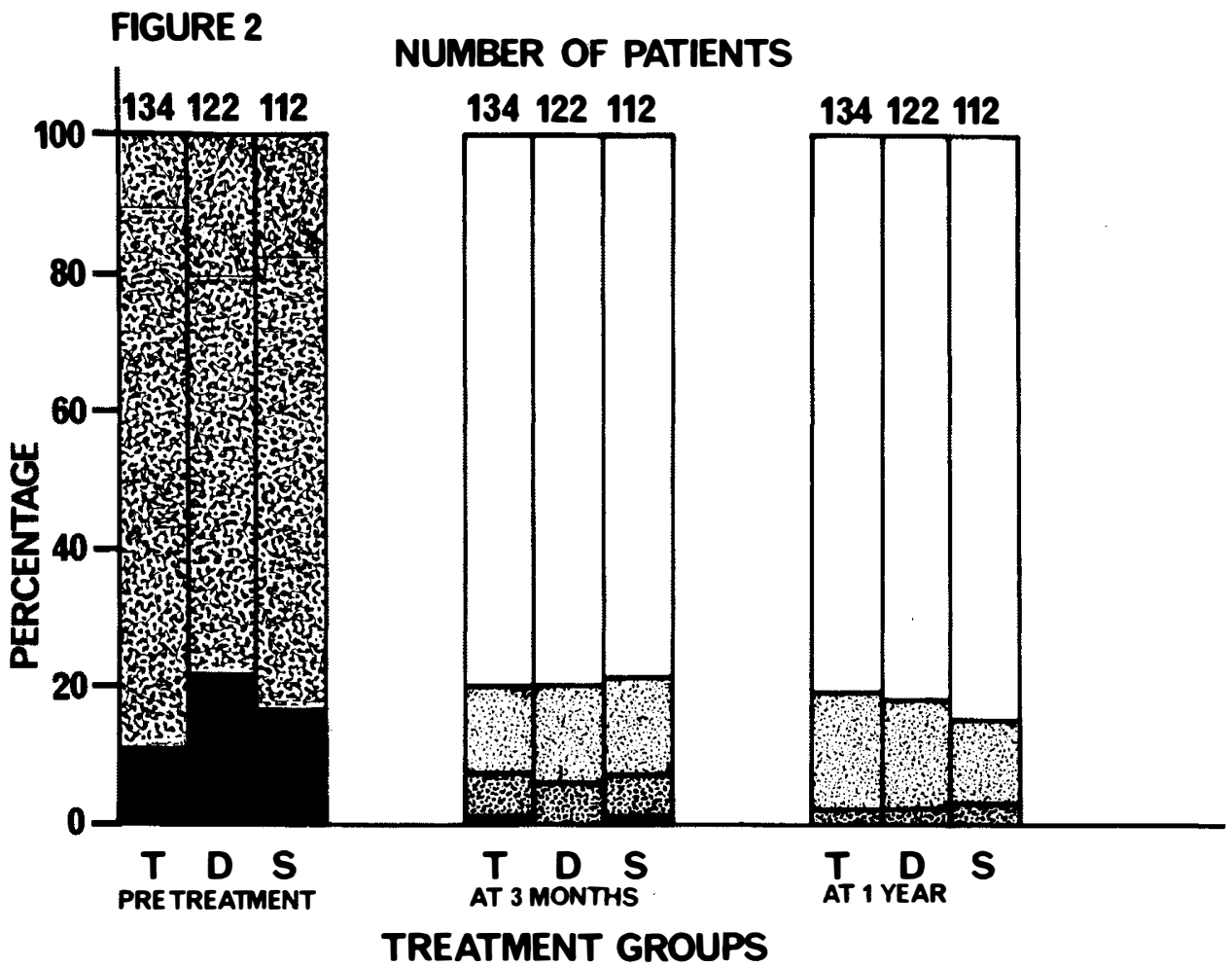

Fig. 2. Changes in the grades of intensity of trachoma in the upper tarsal conjunctiva after intermittent therapy with topical tetracycline ointment $(T)$, oral doxycycline $(D)$, and oral sulphametopyrazine $(S)$, before treatment (left), at 3 months (middle) after, and 1 year (right) after beginning treatment. 
there was no basic medical eye care and treatment. Back-up services in the form of regular mobile eye clinics, were started six months following treatment, and have contributed to maintaining effective eye health care in these villages, particularly in preventing epidemics of bacterial conjunctivitis. These mobile eye clinics were allowed to prescribe only chloromycetin eye drops or ointment for bacterial infections during the oneyear follow-up. A marked reduction in the prevalence of blepharitis was observed at the end of one year in the two villages. Treatment of selected cases of vernal catarrh with sodium cromoglycate may also have played a role in reducing fly-attracting discharges important for the transmission of trachoma.

No observable changes in hygiene or sanitation were noted, and no educational schemes were employed, during the period of the study. The population has access to chemists and it was possible that antibiotics may have been prescribed for systemic disorders.

Difficulties experienced in transporting the conjunctival swabs with transport media negated the cell culture tests. We found a $10 \%$ positivity rate for C.trachomatis in West Bank populations studied concomitantly using the same clinical criteria and employing direct demonstration of the inclusions using an immunofluorescent technique (unpublished data).

We are grateful to Mrs. J. Thomson, Mr. S. Sharif and Mrs. M. Chumbley for their help and support in carrying out this project, and to the Christoffel Blindenmission, Operation Eyesight Universal, and the Order of St. John for financial aid.

We are also grateful to Farmatalia Carlo Erba Ltd for the donation of sulphametopyrazine tablets used in this project; and to the Sub-department of Virology, Institute of Ophthalmology, London, for undertaking the isolation tests.

\section{References}

${ }^{1}$ Dawson DR, Jones BR, Tarrizzo ML: Guide to Trachoma control. World Health Organisation Geneva 1981.

${ }^{2}$ Thomson IM, Chumbley LC: Eye disease in the West Bank and Gaza Strip. Br J Ophthalmol 1984; 68: 598-602.

${ }^{3}$ Dawson CR, Daghfous T, Messadi M, Hoshiwara I, Vastine D, Yoneda C, Schachter J: Severe endemic trachoma in Tunisia. II A controlled therapy trial of topically applied chlortetracycline and erythromycin. Arch Ophthalmol 1974; 92: 198-203.

${ }^{4}$ Darougar S, Jones BR, Viswalingam N, Poirier RH, Allami J, Houshmand A, Farahmandian A, Gibson JA: Family based suppressive intermittent therapy of hyperendemic trachoma with topical oxytetracycline or oral doxycycline. Br J Ophthalmol 1980; 64: 2915.

${ }^{5}$ Darougar S, Jones BR: Trachoma: Br Med Bull 1983; 39: 117-22.

${ }^{6}$ Dawson CR, Jones BR, Darougar S: Blinding and non-blinding trachoma: assessment of intensity of upper tarsal inflammatory disease and disabling lesions. Bull World Health Organ 1975; 52: 279-82.

${ }^{7}$ Darougar S, Treharne JD, Minassian D, ElSheikh H, Dines RJ, Jones BR: Rapid serological test for diagnosis of chlamydial ocular infections. Br J Ophthalmol 1978; 62: 503-8.

8 Jones BR: Laboratory tests for chlamydial infection. Their role in epidemiological studies of trachoma and its control. $\mathrm{Br} J$ Ophthalmol 1974; 58: 438-54.

${ }^{9}$ Jones BR, Darougar S, Mohsenine H, Porier RH. Communicable ophthalmia: the blinding scourge of the Middle East. Br J Ophthalmol 1976; 60: 492-8. 